\title{
Study on Stress Intensity Factor of Two Dimensional Unilateral Crack Based on Finite Element Method
}

\author{
Yi HUANG \\ College of Civil Engineering and Architecture, Guangxi \\ University \\ Guangxi Key Laboratory of Disaster Prevention and \\ Engineering Safety, Guangxi, China \\ Key Laboratory of Disaster Prevention and Structural \\ Safety of Ministry of Education, Guangxi University \\ E-mail: 1980674048@qq.com
}

\author{
Ke WANG \\ College of Civil Engineering and Architecture, Guangxi \\ University \\ Guangxi Key Laboratory of Disaster Prevention and \\ Engineering Safety, Guangxi, China \\ Key Laboratory of Disaster Prevention and Structural \\ Safety of Ministry of Education, Guangxi University \\ E-mail: 595636390@qq.com
}

\author{
Qiao SONG \\ College of Civil Engineering and Architecture, Guangxi \\ University \\ Guangxi Key Laboratory of Disaster Prevention and \\ Engineering Safety, Guangxi, China \\ Key Laboratory of Disaster Prevention and Structural \\ Safety of Ministry of Education, Guangxi University \\ E-mail: 550155230@qq.com
}

\author{
Yanjun CHANG \\ College of Civil Engineering and Architecture, Guangxi \\ University \\ Guangxi Key Laboratory of Disaster Prevention and \\ Engineering Safety, Guangxi, China \\ Key Laboratory of Disaster Prevention and Structural \\ Safety of Ministry of Education, Guangxi University \\ E-mail: yanjun_chang@126.com
}

\begin{abstract}
The relationship between stress, displacement of the crack tip and stress intensity factor is mainly discussed. Calculating K1 of type I crack by finite element software ABAQUS. The effects of different crack forms and the corresponding stress intensity factors on the mesh size are compared. Research results show that: The refinement of the mesh and the optimization of the crack form can improve the accuracy of the calculation results.
\end{abstract}

Keywords-stress intensity factor; finite element method; stress method; crack; displacement method; displacement extrapolation method

\section{INTRODUCTION}

Cracks in structural members will reduce the security of the structural system, even lead to the failure of the entire system. Through the analysis of a large number of fracture accidents, The fracture is related to the existence of defects or cracks in the structure[1]. The failure of the structure or the machine caused by a defect or crack is the most important, the most common failure mode[2]. To determine whether the component can be used safely, the most important is to determine whether the crack will be unstable and extended to lead to the destruction of structure and equipment. According to the viewpoint of fracture mechanics: The stress intensity factor KI of the crack tip is less than the fracture toughness of the material Kc, then the component is safe, otherwise, the component is dangerous [3]Therefore, how to get the stress intensity factor of the crack tip accurately and effectively is always causes the concern of engineering and technical personnel. In the calculation of stress intensity factor, by using the analytic method, the exact solution can be obtained in theory.
However, in engineering practice, Due to the complexity of the structure and the shape of the members and the complexity of the structure stress and the diversity of the crack shape, Analytical methods are often difficult to describe and solve in mathematics. The finite element method is an approximate method of numerical calculation, but because of its powerful modeling function and it can make full use of the computer's computing power, and with the continuous refinement of the grid division, which can continue to be close to the exact solution, So as to meet the needs of engineering practice.

There are many ways to prefabricate crack of the test component. Line cutting crack slot has excellent machining performance, including the better processing efficiency, better machining accuracy and better surface quality which is widely recognized and adopted in the modern manufacturing industry. Because the object of the study of linear elastic fracture mechanics is sharp crack, so, the crack tip of the specimen used for the determination of KIC should be sharp. The sharp crack often made by using of the method of fatigue test. But the fatigue of the prefabricated and fatigue crack is laborious and time consuming, and the length of the crack is not easy to control. It is easy to form stress concentration when the gap is prefabricated.

So in this paper, the main objective is by comparing the calculation results of the stress intensity factor of the slot type crack model and the fatigue crack model to prove that in the case of a certain precision, whether the analysis of the slot type crack model can be used to replace the fatigue crack model. And the second objective is to comparing the accuracy between displacement method and stress method. 


\section{BRIEF INTRODUCTION OF CALCULATION METHOD}

Using ABAQUS to establish the slot type crack model,In the slot type crack finite element model, the stress intensity factor cannot be calculated automatically. So this paper uses two methods of direct calculation of stress intensity factors of crack tip based on the finite element theory, that is, the node displacement method and the nodal stress method:

The theoretical tension stress intensity factor solution of single side crack under uniaxial can be expressed as[4]:

$$
\begin{gathered}
K_{\mathrm{I}}=F \sigma \sqrt{\pi \mathrm{a}} \\
F=1.12-0.23 \frac{a}{b}+10.6\left(\frac{a}{b}\right)^{2} \\
-21.7\left(\frac{a}{b}\right)^{3}+30.4\left(\frac{a}{b}\right)^{4}
\end{gathered}
$$

Displacement method for calculating stress intensity factor[5]:

$$
K_{\mathrm{I}}=\frac{E u_{2} \sqrt{2 \pi}}{4 \sqrt{r}}
$$

Stress method for calculating stress intensity factor:

$$
K_{\mathrm{I}}=\sigma_{y} \sqrt{2 \pi r}
$$

\section{a : length of crack \\ $\mathrm{b}$ : width of model \\ $\sigma:$ stress \\ E: Young's modulus \\ $\mathrm{u}_{2}:$ dispalcement \\ $\mathrm{r}$ : displacement from crack tip}

\section{The FINITE ElEMENT CALCULATION OF StRESS INTENSITY FACTOR}

\section{A. Geometric Dimensions and Basic Parameters of the Example}

Fig. 1 shows a finite large plate with a single side crack, geometric dimensions shown as it: height is $2 \mathrm{~h}=200 \mathrm{~mm}$, width is $2 b=30 \mathrm{~mm}$, crack length is $a=5 \mathrm{~mm}$. Both ends of a flat plate have uniform tensile stress which is $2 \mathrm{MPa}$, Elastic modulus is $\mathrm{E}=57140 \mathrm{MPa}$ (cast iron), Poisson ratio is $v=0.3$.

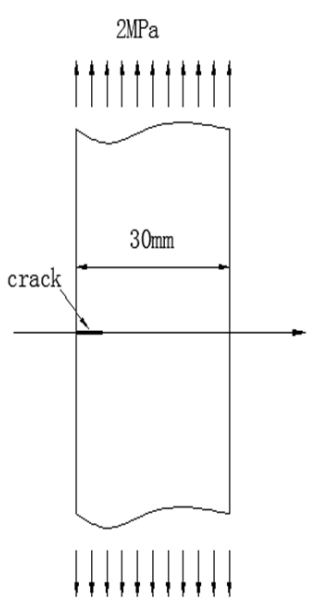

Figure 1. Stress diagrammatic sketc.

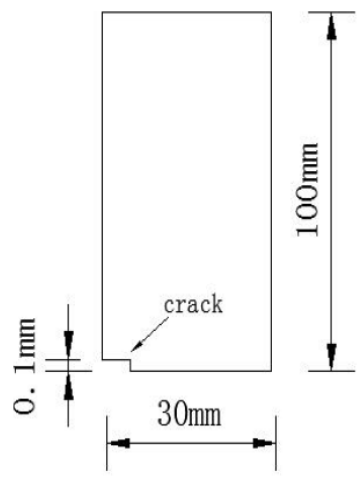

Figure 2. The geometry of Model I with slot crack.

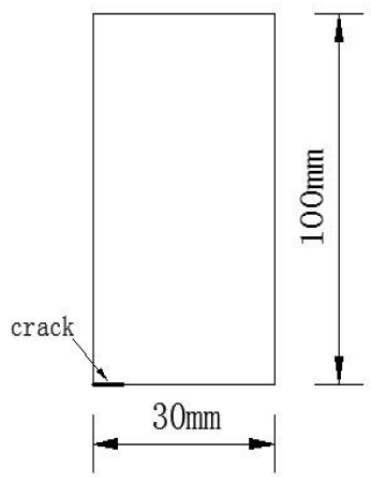

Figure 3. The geometry of Model II with sharp crack

\section{B. Calculating $K_{I}$}

Due to the symmetry of structure and load, so only half of the structure is analyzed in this paper. Fig. 2 is finite element model of slot type crack for opening $0.1 \mathrm{~mm}$, Fig. 3 is the finite element model of the sharp crack with no openings.

Boundary condition:

Lower boundary:Displacement is fixed in vertical 
direction. The crack is free.

Upper boundary:Displacement and rotation are fixed in horizontal direction.

In order to reduce the computational unit, method of grid transition is adopted, at the crack tip up and down $5 \mathrm{~mm}$ at the local location using $0.1 \mathrm{~mm}$ grid, the far field part still uses the big grid.

The model with $0.5 \mathrm{~mm}$ mesh created by Abaqus which is shown in Fig. 4:

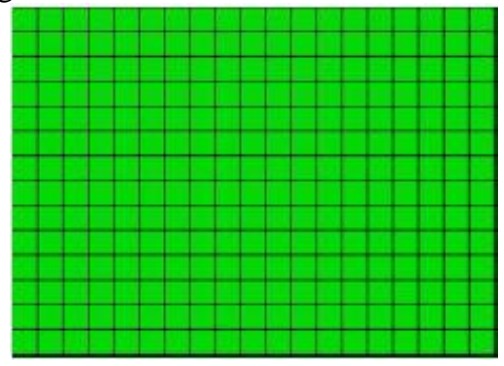

Figure 4. The model with $0.5 \mathrm{~mm}$.

The model with $0.1 \mathrm{~mm}$ mesh created by Abaqus which is shown in Fig. 5:

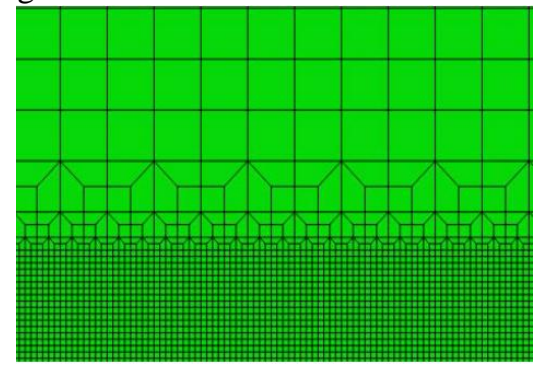

Figure 5. The model with $0.5 \mathrm{~mm}$ mesh.

The displacement method and stress method are used to calculate the stress intensity factor by formula(3) and (4), which will be compared with the theoretical solution calculated by formula(1).

The calculated displacement and stress are plotted in curves, as shown in Fig. 6 and Fig. 7:

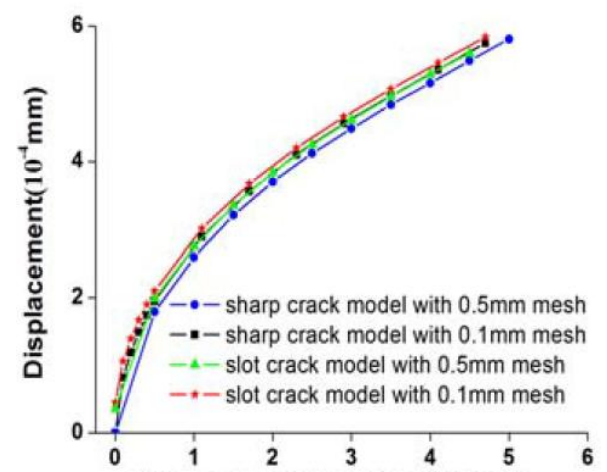

Figure 6. The displacement from crack tip vs. displacement with different mesh of different.

The general trend of displacement curves for each model is the same, we can see that with the refinement of mesh, the displacement is upward.

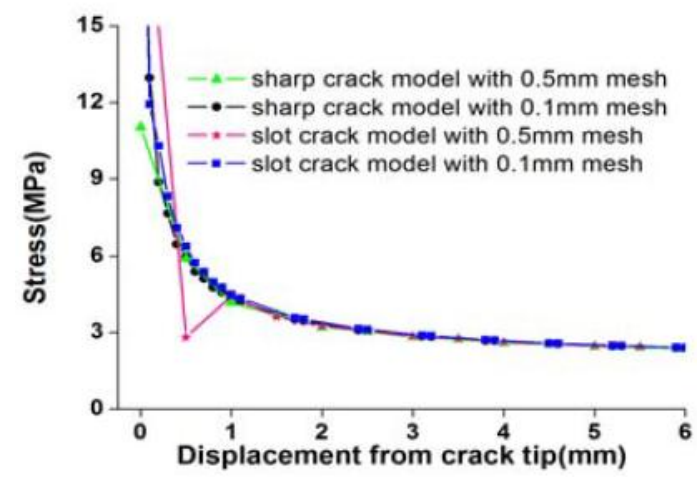

Figure 7. The displacement from crack tip vs. stress with different mesh of different model curve.

Due to the instability at the crack tip, There is a singularity point in the $0.5 \mathrm{~mm}$ grid. The stress gradually stabilized at about $\mathrm{r}=1.5$

The curves of stress intensity factor calculated by displacement method and stress method, drawing curves, are as shown in Fig. 8 and Fig. 9:

By analyzing the result of displacement method, we can get the following conclusions:

The sharp crack model is closer to the theoretical solution than the slot model. In the sharp crack model, the smaller the mesh, the more accurate the solution, but in the slot model, the result is not obvious.

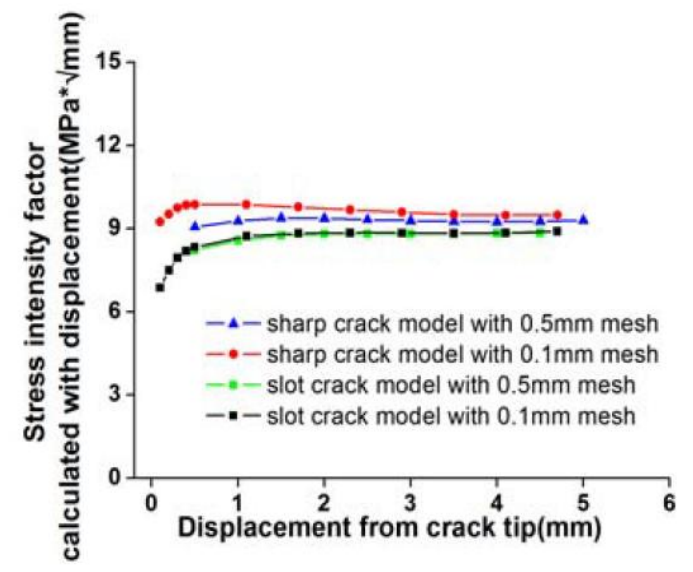

Figure 8. The displacement from crack tip vs. stress intensity factor calculated by displacement with different mesh of different model curve. 


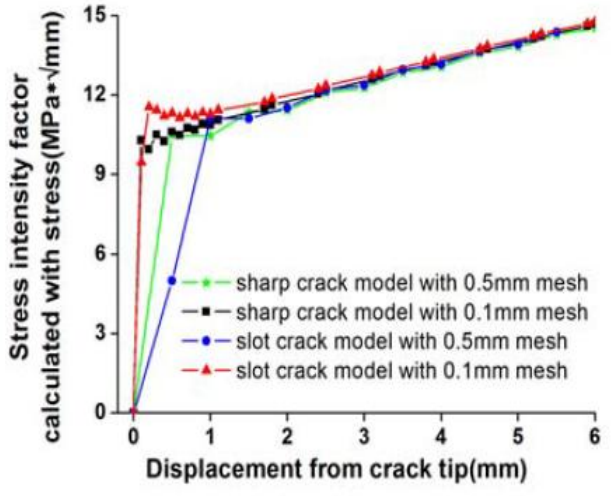

Figure 9. The displacement from crack tip vs. stress intensity factor calculated by stress with different mesh of different model curve.
By analyzing the result of stress method, we can get the following conclusions:

The sharp crack model is closer to the theoretical solution than the slot model.

The calculated results of the model are compared with the theoretical solution derived from the formula (1), The calculated value is close to the theoretical value. So that the models are accurate.

Using the curve which is obtained by the calculation results to fit a smooth curve, using displacement extrapolation method to calculate stress intensity factor in crack tip $(\mathrm{r}=0)$. The predicted stress intensity factors are shown in the tab. 1 :

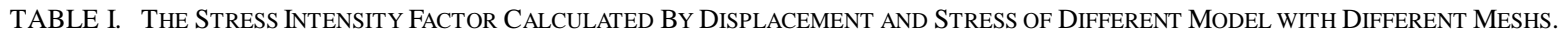

\begin{tabular}{|c|c|c|c|c|}
\hline computing method & grid type of model & $\begin{array}{l}\text { extrapolation method } \\
\mathrm{K} / \mathrm{N}^{*} \mathrm{~mm}^{-3 / 2}\end{array}$ & $\begin{array}{l}\text { Theoretical } \\
\text { solution } \mathrm{K} \\
/ \mathrm{N}^{*} \mathrm{~mm}^{-3 / 2}\end{array}$ & $\begin{array}{c}\text { relative error } \\
\eta(\%)\end{array}$ \\
\hline \multirow{2}{*}{ displacement method } & Model I mesh $0.5 \mathrm{~mm}$ & 8.77 & \multirow{2}{*}{10.30} & 14.85 \\
\hline & Model I mesh $0.1 \mathrm{~mm}$ & 8.85 & & 14.08 \\
\hline & Model II mesh $0.5 \mathrm{~mm}$ & 9.49 & & 7.86 \\
\hline & Model II mesh $0.1 \mathrm{~mm}$ & 10.04 & & 2.52 \\
\hline \multirow{4}{*}{ Stress method } & Model I mesh $0.5 \mathrm{~mm}$ & 9.97 & & 3.20 \\
\hline & Model I mesh0.1mm & 10.54 & & 2.33 \\
\hline & Model II mesh $0.5 \mathrm{~mm}$ & 10.13 & & 1.65 \\
\hline & Model II mesh0.1mm & 10.23 & & 0.68 \\
\hline
\end{tabular}

\section{CONCLUSIONS}

In this paper, the results obtained by two different models of different grids in two kinds of calculation methods are contrasted, the main conclusions are as follows: The two kinds of calculation methods of these two kinds of stress intensity factors all have good performance and high precision. By comparing find that with the refinement of the grid the calculation accuracy is obviously improved The calculation accuracy of the finite element calculation of the sharp crack model (Model II) is also significantly higher than that of the slot type crack model(Model I). It is proved that the finite element method can calculate the stress intensity factor of the complex component and the complicated crack form, with relatively high accuracy, which can be a powerful tool to solve the problem of crack.

\section{ACKNOWLEDGEMENT}

This work was financially supported by the National Natural Science Fund(11262001 and 51465002), the Systematic Project of Guangxi Key Laboratory of Disaster Prevention and Structural Safety(2016ZDX07)and the Guangxi Natural Science Fund (2012GXNSFBAO53145)

\section{REFERENCES}

[1] Qu Weilian, Lu Lijun, Li Ming. Finite element modeling method with three dimensional penetrating crack structure [J]. Journal of Wuhan University of Technology, 2008, 30(1):87-90

[2] Chen Chuanyao. Fatigue and fracture [M]. Wuhan: Publishing House of Huazhong University of Science and Technology,2002.

[3] Chu, Wu Yang. Fracture mechanics based [M]. Science, 1979

[4] China Aviation Research Institute. Stress intensity factor manual [M]. Science Press, 1993

[5] Zhao Shushan Cheng Jin. Fracture mechanics (high school teaching material) [M]. Science Press,2006 